\title{
Vestibular Evoked Myogenic Potentials (VEMP) in patients with Multiple Sclerosis
}

\author{
${ }^{1}$ Gehan Abdel-Rahman El- Zarea, ${ }^{1}$ Ahmed Ismail Abbas, ${ }^{2}$ Mohamed Hamed Rashad, ${ }^{1}$ Ahmed \\ Mohamed Almeciry
}

${ }^{1}$ Department of Audio Vestibular Medicine ENT Department, ${ }^{2}$ Department of Neurology, Faculty of Medicine Al-Azhar University

Corresponding author: Ahmed Mohamed Almeciry, Mobile: 01091249347; email: meciry_a@yahoo.com

\begin{abstract}
Background: multiple sclerosis (MS) is a chronic demyelinating disease in which the insulating covers of nerve cells in the brain and spinal cord are damaged and this damage disrupts the ability of parts of the nervous system to communicate, resulting in a range of signs and symptoms. Objective: this study aimed to assess cervical vestibular evoked myogenic potentials (cVEMP) in patient with multiple sclerosis and to demonstrate the value of cVEMP as a good method for the assessment of vestibulospinal tract in patients with MS. Patients and Methods: to achieve this target, we enrolled 50 subject: 30 patients diagnosed as MS in addition to 20 healthy control. The cVEMP response was evaluated for the presence of positive (p1) and negative (n1) peaks of the first biphasic wave complex; latency ( $\mathrm{p} 1$ and $\mathrm{n} 1$ latency) and peak to peak amplitude. The bilateral otoscopy was normal in cases and controls, with no middle or outer ear disease that could bias the study results being found. The pure tone audiometry showed levels of hearing compatible with normal hearing in the 20 healthy subjects, with hearing thresholds that did not exceed $25 \mathrm{~dB}$ for any individual. Results: the present results showed that $60 \%$ of MS patients had VEMP abnormalities (absent responses and/or prolonged latencies). In the present study, patients with absent VEMP wave were found to have significantly higher disease duration. In the present study, the absence of P13-N23 waves were more frequent in patients with greater EDSS score, and also absence of P13-N23 waves were more frequent in patients with MS with vestibular symptoms and greater disability.

Conclusion: we can say that in patients with MS in which demyelination has extended to the vestibulospinal tract, there is going to be a prolongation of the p13 and n 23 wave. In fact, this increase in the p13 and $\mathrm{n} 23$ latencies is clear in the patients with MS in comparison to the control group.

Keywords: multiple sclerosis, vestibular evoked myogenic potentials, pure tone audiometry, EDSS.
\end{abstract}

\section{INTRODUCTION}

Multiple sclerosis (MS) is a chronic demyelinating disease in which the insulating covers of nerve cells in the brain and spinal cord are damaged and this damage disrupts the ability of parts of the nervous system to communicate, resulting in a range of signs and symptoms. These symptoms include sensory and motor impairment, ataxia, spasticity, fatigue, and cognitive impairment and leads to significant disability ${ }^{(\mathbf{1})}$.

MS was the most frequently seen demyelinating disease, with a prevalence that varies considerably, from high levels in North America and Europe (> 100/100,000 inhabitants) to low rates in Eastern Asia and sub-Saharan Africa (2/100,000 population) ${ }^{(2)}$. A community-based survey in $\mathrm{Al}$ Quseir, Egypt, has found an MS prevalence of $13.74 / 100,000$. A retrospective meta-analysis in different referral centers of Egypt has found 648 patients with definite MS. Vestibular Evoked Myogenic Potentials (VEMP) was a myogenic potential generated by a sound stimulus that allows noninvasive exploration of the vestibular sense organ (saccule and inferior vestibular nerve). VEMP acoustic stimulus can be evoked by presenting clicks through headsets. The macula was activated by sound stimulus, generating an electrical potential which goes through the pathway of the inferior vestibular nerve, lateral vestibular nerve, vestibulospinal tract, and finally, ipsilateral motor neuron of neck muscle (3). Although initially used in peripheral vestibular disorders, recent findings suggested that VEMP reflected functions of the central otolithic pathways as well, indicating that they could also be applied to disorders of the central 
nervous system ${ }^{(4)}$. Evoked potentials play an important role in MS diagnosis due to their ability to detect subclinical lesions ${ }^{(5)}$, while VEMP was one of these evoked potentials. Although this test is a useful and noninvasive method to examine the function of vestibular nerve and inferior brain stem, there are fewer studies evaluated its importance in MS ${ }^{(\boldsymbol{6})}$. Vestibular evoked myogenic potential was a useful diagnostic method in an evaluation of clinically silent lesions in patients with multiple sclerosis (MS), especially in lesions of lower pons and medulla oblongata. Abnormal results of VEMP in patients with MS implicates lesion of the brainstem, despite normal MRI and/or neurological examination ${ }^{(7)}$.Characteristic findings in MS patients were prolonged latencies, similar to as in visual evoked potentials or a nerve conduction block. Several studies investigated cVEMP abnormalities in patients with MS ${ }^{(\boldsymbol{8})}$. However, to the best of our knowledge, cVEMP was not widely used to assess the sacculo colic pathway in diagnosis of neurological diseases affecting vestibular function. So this study was designed to assess the value of cVEMP in MS patients.

\section{AIM of the STUDY}

This study aimed to assess cervical vestibular evoked myogenic potentials (cVEMP) in patient with multiple sclerosis and to demonstrate the value of cVEMP as a method for the assessment of vestibulospinal tract in patients with MS.

\section{SUBJECTS and METHOD}

This was a descriptive cross-sectional study of fifty persons at Audiology Unit ElHussein Hospital during the period from February to August 2018.

Subjects were divided into two groups:

Control group: the control group consisted of 20 volunteer healthy subjects whose mean age was $29.35 \pm 4.38$ years and their age range was 20-36 years.

Inclusion criteria for control group were history of normal neurological development, normal hearing thresholds (threshold level better than or equal to $25 \mathrm{~dB}$ nHL) according to AAOO ${ }^{(9)}$ with no other ontological or neurological disease.

Study group: consisted of 30 patients whose mean age was $30.87 \pm 4.49$ years and their age range was 21-38 years referred from
Neurology Department El-Hussein Hospital. Written consent from all subjects was taken.

Inclusion criteria: definite MS of either relapsing remitting (RR) or secondary progressive (SP) type subjects based on their medical records, neurologist diagnosis according to revised McDonald criteria 2010. Auditory thresholds equal or lower than $25 \mathrm{~dB}$ $\mathrm{HL}$ at all frequencies evaluated (octave frequencies between 0.25 and $8 \mathrm{kHz}$ ), bilaterally. No history of epilepsy, seizures and head injury. No history of any other general diseases.

Exclusion criteria: any degree of hearing loss, limitation of neck rotation, weakness of sternocleidomastoid muscle, consumption of vestibulo toxic or suppressant drugs within the past month.

Equipment: pure tone audiometer model MAICO53, immittancemeter model GSI 39 with a probe tone $226 \mathrm{~Hz}$, Evoked potential Intelligent Hearing system (IHS).

Method: all subjects were submitted to the following: full history taking, otological examination, pure tone audiometry was done at frequencies 250, 500,1000, 2000, 4000 and $8000 \mathrm{~Hz}$. Immitancemetry was performed to exclude otitis media or any middle ear pathology before VEMP testing. Acoustic reflex threshold was done on 500, 1000, 2000 and $4000 \mathrm{~Hz}$ ipsilaterally and contralaterally. Bone conduction was tested at frequencies 500, 1000, 2000 and $4000 \mathrm{~Hz}$. Speech Audiometry also was done to detect the speech reception threshold (SRT) using Arabic Bisyllabic words for adults as shown by Soliman (10) and word recognition scores using Arabic phonetically balanced words Soliman $\boldsymbol{e t}$ al. ${ }^{(11)}$. VEMP was performed. First, the skin was cleansed carefully before application of the electrodes to ensure the impedance is less than $5 \mathrm{k} \mathrm{ohm}$. After cleaning the skin, active surface electrode was placed on the upper half SCM of the stimulated side. Reference electrode and ground electrode were placed on the inner margin of sternoclavicular joint and the forehead, respectively ${ }^{(\mathbf{1 2})}$. The patient was sitting down and turned the chin to the side opposite the stimulus, thereby tightening the sternocleidomastoid muscle ${ }^{(13)}$. For the recording of VEMP response:

Stimulus parameters: broad band click (14). Stimulus repetition rate: $3.10 / \mathrm{sec}$. Stimulus intensity: 88 dBnHL. Polarity: rarefaction Sweeps number: 250 click for both 
traces. Mode of stimulus delivery: through TDH 49 head phones.

Recording parameters: the filter settings: $30-1500 \mathrm{~Hz}$. Time window: $50 \mathrm{msec}$. Gain: $5 \mathrm{KHz}$ gain factor was used.

The intensity at which a clear and repeatable biphasic wave observed was recorded as the VEMP threshold. If no reliable response was obtained, the VEMP threshold was considered as absent. The VEMP amplitude (peak to peak), P13 and N23 latencies was measured at a stimulus level of 88 $\mathrm{dB}$ nHL. Waves were evaluated for being present or absent, and P13 and N23 latencies, P13-N23 amplitude. Positive VEMP is defined as an initial positive polarity (P13), approximately $13 \mathrm{~ms}$ after stimulus onset, and subsequent negative polarity (N23), at nearly $23 \mathrm{~ms}$, giving rise to a biphasic P13-N23 wave. Considering all of this, the parameters of these potentials to be assessed were the presence or absence of response; P13 and N23 wave latency in milliseconds and peak to peak amplitude ratio in $\mu \mathrm{v}$, a very constant parameter that increases in central lesions.

\section{Statistical methods:}

Data were coded and entered using the statistical package SPSS version 25. Data was summarized using mean and standard deviation for quantitative variables and frequencies (number of cases) and relative frequencies (percentages) for categorical variables. Comparisons between groups were done using unpaired t test when comparing 2 groups ${ }^{(15)}$. For comparing categorical data, Chi square $(\chi 2)$ test was performed. Exact test was used instead when the expected frequency is less than $5^{(\mathbf{1 6})}$. $\mathrm{P}$-values less than 0.05 were considered as statistically significant.

\section{RESULTS}

Table 1: comparison between study and control groups as regard air conduction and bone conduction threshold, SRT (dBHL), MCL (dBHL), WR and acoustic reflex threshold in Hz

\begin{tabular}{|c|c|c|c|c|c|c|}
\hline & \multicolumn{4}{|c|}{ Group } & \multirow{3}{*}{$\mathbf{t}$} & \multirow{3}{*}{$P$ value } \\
\hline & \multicolumn{2}{|c|}{ Study group } & \multicolumn{2}{|c|}{ Control group } & & \\
\hline & Mean & SD & Mean & SD & & \\
\hline PTA (AC 250) & 17.50 & \pm 3.74 & 17.87 & \pm 3.74 & -0.491 & 0.624 \\
\hline PTA (AC 500) & 19.67 & \pm 4.10 & 19.62 & \pm 3.99 & 0.050 & 0.960 \\
\hline PTA (AC 1000) & 19.00 & \pm 4.49 & 17.75 & \pm 4.52 & 1.360 & 0.177 \\
\hline PTA (AC 2000) & 16.25 & \pm 4.28 & 16.12 & \pm 4.73 & 0.137 & 0.891 \\
\hline PTA (AC 4000) & 15.75 & \pm 9.29 & 17.75 & \pm 10.62 & -0.996 & 0.322 \\
\hline PTA (AC 8000) & 16.17 & \pm 4.64 & 16.88 & \pm 5.02 & 0.724 & 0.471 \\
\hline BC 500 & 19.58 & \pm 4.15 & 19.50 & \pm 4.05 & -0.051 & 0.960 \\
\hline BC 1000 & 18.67 & \pm 4.59 & 17.25 & \pm 4.52 & 1.521 & 0.132 \\
\hline BC 2000 & 16.17 & \pm 4.35 & 16.00 & \pm 4.83 & 0.179 & 0.858 \\
\hline BC 4000 & 14.67 & \pm 4.86 & 16.12 & \pm 4.87 & -1.469 & 0.145 \\
\hline SRT (dBHL) & 19.33 & \pm 3.62 & 19.37 & \pm 3.79 & -0.055 & 0.956 \\
\hline MCL (dBHL) & 59.17 & \pm 3.81 & 59.13 & \pm 4.06 & 0.052 & 0.959 \\
\hline WR\% & 99.60 & \pm 1.21 & 99.80 & \pm .88 & -0.897 & 0.372 \\
\hline AR (ipsilateral) 500 & 94.75 & \pm 6.00 & 93.38 & \pm 5.82 & 1.137 & 0.258 \\
\hline AR (ipsilateral) 1000 & 96.83 & \pm 5.44 & 97.25 & \pm 5.66 & -0.369 & 0.713 \\
\hline AR (ipsilateral) 2000 & 93.42 & \pm 7.78 & 95.88 & \pm 5.87 & 1.797 & 0.075 \\
\hline AR (ipsilateral) 4000 & 95.33 & \pm 6.44 & 97.38 & \pm 5.88 & 1.608 & 0.111 \\
\hline AR (contralateral)500 & 94.45 & \pm 7.34 & 94.88 & \pm 4.99 & -0.229 & 0.820 \\
\hline AR (contralateral)1000 & 97.05 & \pm 6.87 & 95.88 & \pm 5.17 & 0.648 & 0.520 \\
\hline AR (contralateral)2000 & 103.76 & \pm 5.33 & 95.12 & \pm 5.22 & 5.66 & $<0.001$ \\
\hline AR (contralateral)4000 & 102.23 & \pm 5.76 & 95.25 & \pm 5.67 & 4.829 & 0.001 \\
\hline
\end{tabular}

Table 2 identification of cVEMP among study and control group

\begin{tabular}{|l|c|c|c|c|c|}
\hline \multirow{2}{*}{} & \multirow{2}{*}{ Study } & \multicolumn{3}{|c|}{ Group } \\
\cline { 3 - 6 } & & \multicolumn{2}{|c|}{ Control } \\
\cline { 3 - 6 } & & No & \% & No & \% \\
\hline \multirow{2}{*}{ cVEMP } & Present & 19 & $63.4 \%$ & 20 & $100 \%$ \\
\cline { 2 - 6 } & Abscent & 11 & $36.6 \%$ & 0 & $0 \%$ \\
\hline
\end{tabular}


Table 3: comparison between P13, N23 latencies and (P-P) amplitude ratio at both sides among control group

\begin{tabular}{|c|c|c|c|c|c|c|}
\hline \multirow{2}{*}{} & \multicolumn{4}{|c|}{ Control group } & \multirow{2}{*}{ t } & \multirow{2}{*}{ P value } \\
\cline { 2 - 7 } & \multicolumn{3}{|c|}{ Right } & \multicolumn{2}{|c|}{ Left } & \\
\cline { 2 - 7 } & Mean & SD & Mean & SD & & \\
\hline P13 msec & 11.39 & \pm 1.27 & 11.39 & \pm 1.27 & 0.000 & 1.000 \\
\hline N23 msec & 22.23 & \pm 1.74 & 21.31 & \pm 1.84 & 1.624 & 0.113 \\
\hline Peak to Peak amplitude ratio (P-P) $\mu v$ & 36.63 & \pm 7.66 & 35.10 & \pm 6.92 & 0.663 & 0.511 \\
\hline
\end{tabular}

** No statistically significant difference in P13, N23 latencies and (P-P) amplitude ratio between right and left ear in control group.

Table 4: comparison between P13, N23 latencies and (P-P) amplitude ratio at both sides among Study group

\begin{tabular}{|c|c|c|c|c|c|c|}
\hline & \multicolumn{4}{|c|}{ Study group } & \multirow[t]{3}{*}{$\mathbf{T}$} & \multirow{3}{*}{$\begin{array}{c}P \\
\text { value }\end{array}$} \\
\hline & \multicolumn{2}{|c|}{ Right } & \multicolumn{2}{|c|}{ Left } & & \\
\hline & Mean & SD & Mean & SD & & \\
\hline P13 msec & 13.57 & \pm 2.70 & 13.69 & \pm 2.82 & -0.139 & 0.890 \\
\hline N23 msec & 23.68 & \pm 2.78 & 22.93 & \pm 3.15 & 0.831 & 0.411 \\
\hline Peak to Peak amplitude ratio (P-P) $\mu v$ & 38.31 & \pm 8.77 & 39.25 & \pm 8.35 & -0.357 & 0.723 \\
\hline
\end{tabular}

** No statistically significant difference in P13, N23 latencies and (P-P) amplitude ratio between right and left ear in cases group.

Table 5: comparison between study and control groups as regard P13, N23 latencies and (P-P) amplitude ratio

\begin{tabular}{|l|c|c|c|c|c|c|}
\hline \multirow{2}{*}{} & \multicolumn{2}{|c|}{ Study group } & \multicolumn{2}{c|}{ Control group } & \multirow{2}{*}{ T } & \multirow{2}{*}{ P value } \\
\cline { 2 - 5 } & Mean & SD & Mean & SD & & $* 0.002$ \\
\hline P13 (Rt) msec & 13.57 & \pm 2.70 & 11.89 & \pm 1.27 & 3.466 & $* 0.044$ \\
\hline N23 (Rt) msec & 23.68 & \pm 2.78 & 22.23 & \pm 1.74 & 2.089 & $* 2.505$ \\
\hline $\begin{array}{l}\text { Peak to Peak amplitude } \\
\text { ratio (P-P) Rt } \boldsymbol{\mu v}\end{array}$ & 38.31 & \pm 8.77 & 36.63 & \pm 7.66 & 0.673 & 0.505 \\
\hline P13 (Lt) msec & 13.69 & \pm 2.82 & 11.13 & \pm 1.27 & 3.389 & $* 0.002$ \\
\hline N23 (Lt) msec & 22.93 & \pm 3.15 & 21.31 & \pm 1.84 & 1.996 & 0.055 \\
\hline $\begin{array}{l}\text { Peak to Peak amplitude } \\
\text { ratio (P-P) Lt } \boldsymbol{\mu v}\end{array}$ & 39.25 & \pm 8.35 & 35.10 & \pm 6.92 & 1.714 & 0.095 \\
\hline
\end{tabular}

** It shows highly statistically significant difference between study and control groups as regard P13 and $\mathrm{N} 23$ latencies in both right and left ear but no statistically significant difference was observed as regard (P-P) amplitude ratio.

Table 6: effect of gender on P13, N23 latencies and (P-P) amplitude ratio in the study group

\begin{tabular}{|c|c|c|c|c|c|c|}
\hline & \multicolumn{4}{|c|}{ Sex } & \multirow{3}{*}{$\mathbf{t}$} & \multirow{3}{*}{ P value } \\
\hline & \multicolumn{2}{|c|}{ Male } & \multicolumn{2}{|c|}{ Female } & & \\
\hline & Mean & $\begin{array}{l}\text { Standard } \\
\text { Deviation }\end{array}$ & Mean & $\begin{array}{l}\text { Standard } \\
\text { Deviation }\end{array}$ & & \\
\hline P13 & 12.54 & 1.43 & 13.93 & 2.97 & -1.089 & 0.289 \\
\hline $\mathbf{N 2 3}$ & 21.54 & 2.00 & 24.44 & 2.65 & -2.435 & 0.024 \\
\hline $\begin{array}{c}\text { Peak to Peak } \\
\text { ratio (P-P) }\end{array}$ & 39.87 & 4.94 & 37.76 & 9.85 & 0.496 & 0.625 \\
\hline
\end{tabular}

** No statistically significant difference in P13, N23 latencies and (P-P) ratio among both males and females in the study group.

Table 7: comparison between patient's subgroup according to presence of presenting symptoms as regard P13, N23 latencies and (P-P) amplitude ratio

\begin{tabular}{|c|c|c|c|c|c|c|c|c|c|c|c|c|}
\hline & \multicolumn{10}{|c|}{ Presenting symptoms } & \multirow{3}{*}{$\mathbf{F}$} & \multirow{3}{*}{$\begin{array}{c}\mathbf{P} \\
\text { value }\end{array}$} \\
\hline & \multicolumn{2}{|c|}{$\begin{array}{l}\text { bluring of } \\
\text { vision }\end{array}$} & \multicolumn{2}{|c|}{$\begin{array}{c}\text { gait } \\
\text { disturbance }\end{array}$} & \multicolumn{2}{|c|}{ Headache } & \multicolumn{2}{|c|}{$\begin{array}{c}\text { rt LL } \\
\text { weakness }\end{array}$} & \multicolumn{2}{|c|}{$\begin{array}{c}\text { weakness of } \\
\text { both } L L\end{array}$} & & \\
\hline & Mean & SD & Mean & SD & Mean & SD & Mean & SD & Mean & SD & & \\
\hline P13 & 14.42 & 4.01 & 12.02 & 1.20 & 14.79 & 2.37 & 12.72 & 2.91 & 14.01 & 2.82 & 0.954 & 0.456 \\
\hline $\mathbf{N 2 3}$ & 22.92 & 2.85 & 23.21 & 3.32 & 24.47 & 1.91 & 22.60 & 3.63 & 25.72 & 1.49 & 0.797 & $\overline{0.543}$ \\
\hline $\begin{array}{c}\text { Peak to } \\
\text { Peak ratio } \\
(\text { P-P) }\end{array}$ & 40.28 & 7.73 & 40.80 & 6.94 & 38.33 & 12.82 & 36.22 & 7.58 & 35.00 & 9.17 & 0.286 & 0.883 \\
\hline
\end{tabular}

** No statistically significant difference in P13, N23 and (P-P) ratio among different presenting symptoms in the study group. 
Table 8: comparison between patient's subgroup according to presence of dizziness as a complaint as regard P13, N23 latencies and (P-P) amplitude ratio

\begin{tabular}{|c|c|c|c|c|c|c|}
\hline & \multicolumn{4}{|c|}{ Dizziness } & & T \\
\hline & \multicolumn{2}{|c|}{ present } & \multicolumn{2}{c|}{ Absent } & Palue \\
\hline & Mean & SD & Mean & SD & & \\
\hline P13 msec & 17.25 & \pm 2.03 & 12.55 & \pm 1.84 & 4.957 & $<0.001$ \\
\hline N23 msec & 23.22 & \pm 3.23 & 23.81 & \pm 2.73 & -0.414 & 0.683 \\
\hline $\begin{array}{c}\text { Peak to Peak amplitude } \\
\text { ratio (P-P) } \boldsymbol{\mu v}\end{array}$ & 36.02 & \pm 9.27 & 38.95 & \pm 8.80 & -0.651 & 0.522 \\
\hline
\end{tabular}

** It shows highly statistically significant difference among study group as regard presence and absence of dizziness.

Table 9: comparison between patient's subgroup according to EDSS score as regard P13, N23 latencies and (P-P) amplitude ratio

\begin{tabular}{|c|c|c|c|c|c|c|}
\hline & \multicolumn{4}{|c|}{ EDSS } & & \\
\hline & \multicolumn{2}{|c|}{$>5.5$} & \multicolumn{2}{c|}{ (5.5 } & P value \\
\hline & Mean & $\begin{array}{c}\text { Standard } \\
\text { Deviation }\end{array}$ & Mean & $\begin{array}{c}\text { Standard } \\
\text { Deviation }\end{array}$ & & \\
\hline P13 & 15.35 & 1.64 & 13.30 & 2.75 & 1.242 & 0.228 \\
\hline N23 & 25.15 & 1.48 & 23.46 & 2.88 & 0.980 & 0.338 \\
\hline $\begin{array}{c}\text { Peak to Peak ratio (P- } \\
\text { P) }\end{array}$ & 42.00 & 16.46 & 37.76 & 7.62 & $\mathbf{0 . 4 3 9}$ & $\mathbf{0 . 7 0 1}$ \\
\hline
\end{tabular}

** It shows no statistically significant difference as regard P13, N23 latencies and (P-P) amplitude ratio and EDSS score.

\section{DISCUSSION}

The present study aimed to assess cervical vestibular evoked myogenic potentials (cVEMP) in patient with MS and to demonstrate the value of cVEMP as a good method for the assessment of vestibulospinal tract in patients with MS.

To achieve this target, we enrolled 50 subjects: 30 patients diagnosed as MS in addition to 20 healthy control. In MS patients, relations were evaluated between VEMP waves and presenting symptoms, including vestibular symptoms, presence of a demyelinating lesion in the brainstem on MRI, disease duration and EDSS score.

In our study, the multiple sclerosis patients had mean age of $30.87 \pm 4$.49years and their age range was 21-38 years, while the control group had a mean age of $29.35 \pm 4.38$ years and their age range were 20-36 years without statistically significant difference between both groups and this was in agreement with most epidemiological studies on the age of onset of MS.

Gracia et al. (17) reported that there were various studies that have been carried out on this disease and in all of them the fact that the first symptoms generally appeared between the ages of 20 and 40 years. Sharafaddinzadeh et al. ${ }^{(18)}$ reported that the mean age of MS onset was in the third decade from 25.8 to 30.9 years.
The peak age of onset is approximately 5 years earlier for women than for men.

Harirchian et al. ${ }^{(12)}$ reported in their study that the mean age MS patients: 20-40 years; mean age: $30 \pm 5.4$ years. Milo and Kahana ${ }^{(19)}$ reported that MS usually appears in adults in their late twenties or early thirties but it can rarely star $\mathrm{t}$ in childhood and after $50 \mathrm{y}$ ears of age. All subjects in the study and the control groups had pure tone thresholds equal or less than $25 \mathrm{~dB}$ HL for all tested frequencies $(0.25 \mathrm{KHz}$ through $8 \mathrm{KHz})$. This indicated normal peripheral hearing according to AAOO (9) with no significant difference between both groups. Our study showed that, speech audiometry revealed that speech reception threshold (SRT) is in agreement with pure-tone average for all subjects and word discrimination scores (WDS) were excellent with no significant difference between the control and study groups. In our study we found that normal middle نقول ear functions were observed in all subjects, as shown by acoustic immittance test results (all subjects had type A tympanogram). All ears had acoustic reflex thresholds within normal range when elicited ipsilaterally with no significant difference between the control and study groups, but according to contralateral acoustic reflex thresholds there was significant 
difference between the control and study group.Hearing loss was un common symptoms of MS, about $4 \%$ to $10 \%$ of patients diagnosed with MS develop sensory-neural hearing loss ${ }^{(20)}$.Tekin et al. ${ }^{(21)}$ reported that in $4-10 \%$ of the MS patients, sensorineural hearing loss occurred between relapses or remissions. Zeigelboim et al. ${ }^{(22)}$ found no evidence of hearing loss in MS patients. Nevertheless, regardless of the age range, affected patients were able to respond to every tested frequency. In this study, the presenting symptoms in our patients were as follows: weakness of lower limb extremities (10 cases), gait disturbance (9 cases), headache (6 cases) and blurring of vision (5 cases). On the other hand, dizziness and balance impairment as a complaint were present in 12 (40\%) of MS patients and this was in agreement with. Susan and Jesse (23) reported that over $90 \%$ of people with MS report mobility difficulties, and maintaining mobility is consistently ranked as one of the highest priorities for this group, independent of disease duration or disability level.

In this study we found that the mean overall p13 wave latency in in the total sample of MS cases was 13.57 and $13.69 \mathrm{msec}$ for right and left ear respectively. If compared with that of the control group, in which the mean p13 wave latency was 11.89 and $11.13 \mathrm{msec}$ for right and left ear respectively, it showed that there is a statistically significant increase in the p13 wave latency in our patients with MS.

We also found that the overall mean N23 wave latency for right ears of MS group was $23.68 \mathrm{msec}$. Again, if it compared with that obtained in the control group in which the mean $\mathrm{N} 23$ wave latency for right ears was $22.23 \mathrm{msec}$, it showed that there is a statistically significant increase in the $\mathrm{n} 23$ wave latency in patients with MS.

Gracia et al. (17) reported that prolonged latencies in MS patients were the most common findings which may be attributed to reduced conduction velocity because of demyelination of vestibulospinal tract axons or primary afferent axons in the nerve root entry zone and absence of cVEMP waves in MS patients may be attributed to advanced damage to the myelin sheath or axonal degeneration. Although demyelinating lesions typically cause reduced conduction, severe demyelination may cause conduction block, desynchronized conduction and phase loss which may result in loss of response. Axonal degeneration and demyelination in MS may begin early in the disease course and progress, they also reported that in patients with MS in which demyelination had extended to the vestibulospinal tract, there is going to be a prolongation of the p13 and $\mathrm{n} 23$ wave latencies. In fact, this increase was statistically significant in the p13 and n23 latencies was clear in the patients with MS in comparison to the control group, also the affectation of both ears has also been confirmed in their study. Harirchian et al. ${ }^{(12)}$ showed that $70 \%$ of MS patients had some form of abnormality and the mean abnormality in their study was prolongation of $\mathrm{P} 13$ and N23 latencies in MS patients, and therefore Vestibular evoked myogenic potential has a high sensitivity (70\%) in MS patients.Varied results had been reported previously about the relation between cVEMP abnormalities, clinical signs and presence of brainstem lesions on MRI scans.In this study there was no statistically significant difference between cVEMP abnormalities and clinical signs or presence of brainstem lesions on MRI scans and this was in agreement with results of Garcia $\boldsymbol{e t}$ al. (17) who found increased p13 and n 23 latencies. However, a correlation with the clinical findings and abnormal cVEMP responses was not present; patients with clinical signs and symptoms and abundant MRI lesions had normal cVEMP results whereas asymptomatic patients with few lesions on imaging showed greatly altered potentials.Also, Garcia et al. ${ }^{(17)}$ were also unable to find a correlation between VEMPs and brainstem clinical or MRI lesions. According to the above-mentioned data, testing cVEMPs in MS cases is not a sensitive way of documenting brainstem involvement and this agrees with results of Gracia et al. ${ }^{(17)}$ who showed that absence of correlation between the results for the potentials and clinical signs or symptoms or the characteristic radiological alterations of MS. In the current study, abnormal cVEMP response was more frequent in ears of MS patients with vestibular symptoms than without and in patients with great EDSS score. This is in agreement with those of Koura and Hessein $^{(4)}$ who reported that absence of P13N23 waves was more frequent in patients with higher EDSS score and also absence of P13N23 waves was more frequent in MS cases with vestibular symptoms. Also, Guven et al. ${ }^{(24)}$ reported that absence of $\mathrm{p} 1-\mathrm{n} 1$ waves was more frequent in ears of MS cases with vestibular 
symptoms and also more frequent in ears of MS patients with greater EDSS score.

Also, Gazioglu and Boz ${ }^{(25)}$ studied both ocular and cervical VEMPs and had found that $n 1$ latency of ocular vestibular-evoked myogenic potentials (oVEMPs) and p1 latency of cVEMP were prolonged in MS patients and were significantly correlated with EDSS. The mean duration of disease was $7.90 \pm 3.34$ and this study showed that there was no statistically significant difference between VEMP abnormalities and disease duration and this was in agreement with Gazioglu and Boz ${ }^{(25)}$ who reported that cVEMP abnormalities were not correlated with disease duration and Harirchian et al. ${ }^{(12)}$ who showed that there was no statistically significant relation between a course of the disease and abnormal cVEMP. As regard amplitude, data on amplitudes provide less information, without being able to reach a plausible conclusion. As amplitude is one of the most variable VEMP parameters, it was influenced by many extrinsic factors and appears altered in both middle ear disease and in central and peripheral lesions. In contrast, according to the other cVEMP parameter peak to peak ratio (P-P ratio) the mean value of (P-P ratio) for MS cases was 38,31 $\mu \mathrm{v}$ and $39.25 \mu \mathrm{v}$ for both right and left ear consequently, while that of control group was $36.63 \mu \mathrm{v}$ and 35.10 $\mu \mathrm{v}$ for both right and left ear consequently with no statistically significant differences between MS cases and control group.

This is in agreement with results of Koura and Hussein ${ }^{(4)}$. They reported that there were no statistically significant differences between patients with preserved cVEMP and controls in the mean value of cVEMP amplitude or interaural amplitude ratio in right or left side. Guven et al. ${ }^{\text {(24) }}$ showed that the mean $\mathrm{p} 1-\mathrm{n} 1$ amplitude was lower in the MS cases than in control groups but there were no significant differences between MS and control group in mean $\mathrm{p} 1, \mathrm{n} 1$. Harirchian et al. ${ }^{(12)}$ showed the variability of the amplitude can be due to the patient position, lack of cooperation, and inability of the subject to keep the SCM muscle tonically contracted for a few seconds. Therefore, they suggested that amplitude should not be used as criterion to define the VEMPs as normal or pathological. Gracia et al. (17) also reported that the inter-aural difference in amplitude was also evaluated in all the patients. However, the results obtained for this factor showed a clear disparity and were not taken into account in the statistical study.

Finally, the assessment of VEMP could be useful in detecting subclinical lesions or evaluation of vestibulospinal pathway in MS patients.

\section{CONCLUSION}

cVEMP is a quick, simple, painless and complementary with other neurophysiological and radiological tests in assessing brainstem lesions in MS.

cVEMP is not a sensitive way to decument brainstem involvement in MS patients (only $60 \%$ of MS patients had VEMP abnormalities depending on vestibulospinal tract affection by demylination process.Abnormal VEMP waves were more frequent in MS cases with vestibular symptoms, longer disease duration and greater disability (EDSS).No correlation between cVEMP abnormalities and clinical signs and MRI finding of brain involvement in MS patients.

\section{REFERENCES}

1. Fissolo N, Montalban $X$ and Comabella $M$ (2016): DNA vaccination techniques. Multiple Sclerosis Methods and Protocols, 1304: 39-50.

2. Leray E, Moreau T, Fromont $A$ and Edan G (2016): Epidemiology of multiple sclerosis. Neuroepidemiology, 172(1):313.

3. Koura $R$ and Hussein $M$ (2018): Vestibular-evoked myogenic potential: an easy neurophysiological tool for evaluating brain stem involvement in multiple sclerosis The Egyptian Journal of Otolaryngology, 34:144-148.

4. Venhovens $\mathbf{J}$, Meulstee $\mathbf{J}$ and Verhagen (2016): Vestibular evoked myogenic potentials (VEMPs) in central neurological disorders, Clin. Neurophysiol., 127: 40-49.

5. Patkó T, Simó $M$ and Arányi $Z$ (2007): Vestibular click-evoked myogenic potentials: sensitivity and factors determining abnormality in patients with multiple sclerosis. Mult. Scler., 13:193201.

6. Akin $F$ and Muronane $O$ (2003): The effects of click and tone burst stimulus parameter on the VEMP. Am. Acad.Audiol., 14:500-506.

7. Eleftheriadou A, Deftereos S, Zarikas $\mathbf{V}$ et al. (2009): The diagnostic value of 
earlier and later components of vestibular evoked myogenic potentials (VEMP) in multiple sclerosis. J. Vestib. Res., 19:5966.

8. Rosengren $S$ and Colebatch J (2011): Ocular vestibular evoked myogenic potentials are abnormal in internuclear ophthalmoplegia. Clin. Neurophysiol., 1264-1267.

9. American Academy of Ophthalmology and Otolaryngology (1979): American academy of ophthalmology and otolaryngology committee on hearing and equilibrium and the american council of otolaryngology committee on the medical aspects of noise. Guide for the evaluation of hearing handicap. JAMA., 241:2055-2059

10. Soliman S (1976): Speech discrimination audiometry using arabic phonetically balanced words. Ain Shams Medical Journal, 27: 27-30.

11. Soliman S, Fathalla A and Shehata W (1985): Development of the arabic staggered spondaic words (SSW) Test. Proceedings of the $8^{\text {th }}$ Annual Ain Shams Congress, 2:1220-1246.

12. Harirchian $M$, Karimi $N$, Nafisi $S$, Akrami S, Ghanbar-ian D and Gharibzadeh S (2013): Vestibular evoked myogenic potential for diagnoses of multiple sclerosis: Is it beneficial? Med. Glas. (Zenica),10(2):321-326

13. Huang T, Cheng $P$ and Su $H$ (2006): The influence of unilateral versus bilateral clicks on the vestibular-evoked myogenic potentials. Otol. Neurotol., 27: 193-196.

14. Burkard $R$ and Shepard N (2013): Vestibular evoked myogenic potentials: They are the same as an auditory evoked potential, only different.Mayo. Clinic, 26: 1125.

15. Chan YH (2003a): Biostatistics102: quantitative data parametric \& nonparametric tests. Singapore Med. J., 44(8): 391-396.
16. Chan YH (2003b): Biostatistics 103: qualitative data-tests of independence. Singapore Med. J., 44(10): 498-503.

17. Garcia V, Carratala I, Alborch $M$ et al. (2013): Vestibular evoked myogenic potential findings in multiple sclerosis. Acta Otorrinolaringol. Esp., 64(5):352-358

18. Sharafaddinzadeh $N$, Moghtaderi $A$, Majdinasab $\mathrm{N}$ et al. (2013): The influence of ethnicity on the characteristics of multiple sclerosis: a local population study between Persians and Arabs. Clin. Neurol. Neurosurg., 115(8):1271-1276.

19. Milo $R$ and Kahana E (2010): Multiple sclerosis: geoepidemiology, genetics and the environment. Autoimmunity Reviews, 9 (5): 387-394.

20. Peyvandi A, Naghibzadeh $B$ and Roozbahany N (2010): Neuro-otologic manifestations of multiple sclerosis. Arch Iran Med., 13(3):188-192.

21. Tekin M, Acar G, Cam $O$ and Hanege $F$ (2014): Sudden sensorineural hearing loss in a multiple sclerosis case. North Clin. Istanb., 1(2): 109-113.

22. Zeigelboim B, Arruda W, Iório $M$, Jurkiewicz A,1 Bassetto J, Klagenberg K and Albernaz $P$ (2007): High-frequency hearing threshold in adult women with multiple sclerosis. International Tinnitus Journal, 13(1):11-14.

23. Susan $L$ and Jesse V (2014): Understanding and treating balance impairment in multiple sclerosis from the department of rehabilitation and movement science. $\mathrm{J}$ of University of Vermont, Burlington, 21(9): 419-432.

24. Guven H, Bayir O, Aytac E, Ozdek A, Comoglu S and Korkmaz H (2013): Vestibular-evoked myogenic potentials, clinical evaluation, and imaging findings in multiple sclerosis Neurol. Sci., 35:221226.

25. Gazioglu S and Boz C (2012): Ocular and cervical vestibular evoked myogenic potentials in multiple sclerosis patients. Clin. Neurophysiol., 123:1872-1879. 